\title{
Urgences
}

\section{Temps tout terrain}

Denuis Saint-Yves

Numéro 7, 2e trimestre 1983

URI : https://id.erudit.org/iderudit/025108ar

DOI : https://doi.org/10.7202/025108ar

Aller au sommaire du numéro

Éditeur(s)

Urgences

ISSN

0226-9554 (imprimé)

1927-3924 (numérique)

Découvrir la revue

Citer ce document

Saint-Yves, D. (1983). Temps tout terrain. Urgences, (7), 75-82.

https://doi.org/10.7202/025108ar

Ce document est protégé par la loi sur le droit d'auteur. L’utilisation des services d'Érudit (y compris la reproduction) est assujettie à sa politique d'utilisation que vous pouvez consulter en ligne.

https://apropos.erudit.org/fr/usagers/politique-dutilisation/
Cet article est diffusé et préservé par Érudit.

Érudit est un consortium interuniversitaire sans but lucratif composé de l’Université de Montréal, l'Université Laval et l'Université du Québec à Montréal. Il a pour mission la promotion et la valorisation de la recherche. https://www.erudit.org/fr/ 


\section{DENUIS ST-YVES}

\section{Temps tout terrain}




\section{BELLE}

Je te plumerai, nomade exquise. Un jour, tu prendras la relève du temps. Ceci dit, quelle heure est-il? Ici ou là, là-bas. L'alphabet à peine expliqué, la cadence mène au doute. Les syllabes sont muettes cet été, sèchent sur ta peau. Descends de l'escalier, un peu, que j'imagine autre chose. Ne te repose pas, surtout, toute sommation vient du jour.

Rien à déclarer?

Les oiseaux vont par deux comme au cinéma, je relève des traces de toi sur le calendrier, Dieu sait où il va. Moi pas. La nuit met un terme à la discussion où voir et penser est impossible, dissident.

Tout est là, dans les phares, le jour, la nuit et la bague que tu me rends. J'avance avec toi mais à reculons, c'est si loin chez toi. Ne regarde pas en arrière, les recours didactiques c'est pour les cons, la galerie.

L'aile pour muse je n'y pensais plus, c'est joli. Tu penseras à moi souvent, là-bas, dis? Non. Les pistes ça s'invente. J'ai peur de l'abstraction comme de la mort. Sans courir, je changerai les draps du lit, la serrure. Tout mon vocabulaire. Quand pars-tu? Je rentre.

Je n'ai pas tes secrets. Le vinaigre c'est pas moi à la fin. Je ne veux pas de ce poème qui raconte autre chose que la vérité. Tu es belle ce soir, je t'amène danser comme autrefois. Ta robe toujours la même roule sur la piste. La plume dans tes cheveux c'est une plume d'alouette, j'attendais pour te le dire. C'est un cliché idiot, je sais, $i$ am sorry comme tu dis. A cette heure je t'aime. Cliché sur cliché que tu dis. À tout hasard, ne me quitte pas.

Impossible de dire qui de nous deux met du temps à dire adieu à l'autre. Même que c'est amusant, nous nous regardons sans savoir ce qui se passe. Les paroles s'amenuisent petit à petit, comme nous. Dis, c'est pas I'heure de lire The Future of Man. II me déclube drôlement Teilhard de Chardin, on ne badine pas avec la création. Ce ticket entre tes mains épuise le présent. Aucun 
sens de l'orientation non aucun, aucun. Pour où? La cage c'est selon. Là-bas, tu recommenceras à parler.

Une cigarette? Là-bas mais où? La solitude c'est image par image que ca se déroule. Les oiseaux vont te regretter. Beau cliché. Gros rire malgré la douleur. Un événement à l'amiable te convient et j'en ai la chair d'oiseau. Belle, auras-tu la précision de tirer à cóté?

II pleut, le pare-brise tient par miracle. Je con, les essuie-glaces font la gueule. Ne ris pas, c'est moi qui rame. Où sommes-nous? II vaudrait mieux arrêter. Tu me soupçonnes de vouloir t'embrasser, et je n'y peux rien. Tiens, là, sur la gauche, un peu plus loin. L'été c'est l'été mais c'est pas sûr et je sais que tu prends du-retard. Embrasse-moi, sur la bouche. La joue mais non, dis?

Roméo et Juliette, c'est bien loin. Je me souviens qu'on cadrait bien dans ce style-là. Si seulement,

\section{si seulement}

je savais parler aux femmes comme Ferland, mais si ne fait pas fortune. Je préfère purger ma peine en commençant par le début. II pleut par le toit j'en ai conscience, je te dis que c'est la mode et nous passons sur la banquette arrière.

A la radio, nécessité d'une mise au point parfaite: de la salade verte, rouge, noire. Autre cliché pour essayer quelque chose. Stars on long play, c'est déjà mieux. Puis Vol de nuit de Céline Lomez c'est joli, ça te grapille un instant. Tes nylons débusqués prennent la fuite, je te remonte. Tu erres quand tu me donnes la main. Tu serres. Ton corps je le vois. C'est un nid, là-bas.

Un nid, dis? Je ne pensais pas à l'oeuf je m'excuse je je je $i$ am sorry comme tu dis. Ferme tes yeux je ne parlerai pus.

La pluie mais où est-elle passée? La vilaine. Je te suis des yeux pour voir ce que tu vas décider. J'ai tout mon temps. 
A l'aéroport tu suis d'inlassables escaliers qui se font un plaisir de marcher pour toi, tu suis un monde qui n'est pas le mien. Tu t'énerves, bouscules une vieille dame sans t'en apercevoir. On annonce un départ puis un autre, blablabla. Ton pas, dis c'est une flèche, ca me traverse le coeur. Est plate mais c'est tout ce que je trouve à te dire. Je sais, l'avion ça décolle à heure fixe, pas le temps de se cajoler une dernière fois. La barrière nous sépare et seul le geste de la main peut encore quelque chose. Je ne fais pas exprès pour pleurer $i$ am sorry comme tu dis. Tu te retournes une dernière fois, et c'est pour me dire quelque chose que je ne comprends pas. Je fais comme si. Oui, oui, le temps arrange tout à force de faire oui de la tête. Je t'aime et je prendrai soin des oiseaux comme autrefois. 


\section{MUSIC MY LOVE}

Des préoccupations se font sentir là où il n'y a qu'à regarder. Le parc, la verdure, un vieil air de Lovin' Spoonful, Summer in the City or Daydream? C'est ça ou Turn! turn! turn! the Byrds, dans la tête. On annonce de la pluie pour la fin de la journée, rien ne presse et ne me quitte pas trop vite, tu arrives à peine. Le temps attendra bien un peu. Tu fredonnes Get back, et Here comes the sun. Tout bas. Surchauffés de music, imaginant le pire, nous restons là, le corps renversé.

Le tourisme se fait rare, pourtant l'été 81 s'annonce chaud, sans trop de pluie. À Percé, on dit que le tourisme préfère les plages de Floride. Le rocher n'invite plus à la récréation comme autrefois même s'il coûte moins cher que le stade olympique. On entend Stars on long play partout. Les oiseaux s'habituent et nous filons le parfait bonheur. Music my love, tu fais Toulouse, Streisand, Coolidge, Benatar, Reno, Tucker, enfin, des trucs fous, qui se passent de commentaire et continuent à préciser. Ta scène, c'est moi, ne me quitte pas.

Midi. Des tranches de ma vie, d'orange, défilent dans ta bouche. Tu parles la bouche pleine, et tu m'aimes. Diane Tell, ça te dit quelque chose? En flèche, tu le connais par coeur. Dis donc! Magic music. Le train mélange un peu la conversation, des yeux en sortent, tu les dévores de curiosité. On dirait Bruce Cockburn de profil, là-bas, ce n'est qu'un mirage. Avec les artistes, on ne sait jamais. Le train s'évanouit, et tu reviens à Diane Tell.

Love you live, the Rolling Stones à Toronto, et Mick. Sur invitation seulement. Sympathy for the devil, comme un chapelet à la percussion un peu lourde dans ton chagrin. My love, la mer est belle, n'y pensons plus. II ne manque que les Beach Boys. Gros rires. Le temps file droit devant lui, les yeux dans les barques, qui tapissent la mer. Tu voudrais construire un château avec du sable mais Duke Ellington's ne viendra pas. En pure perte.

Je glisse dans la conversation le nom de Léo Ferré et tu t'en empares comme d'un fruit. Le sable est fin et tes pieds y moulent des empreintes de coquillages. Autour de nous des passants vont 
et viennent sans faire attention à tes seins nus. Je les embrasse. Léo Ferré aussi.

Vois-tu, là-bas, l'affiche c'est Offenbach. Tu aimes, mais préfères Francoeur. C'est un jeu électronique, un juke-box péremptoire qui sort avec les filles, qui reçoit des pièces de cuir. Son Nelligan te remonte à la gorge comme une messe noire. II est perforé d'un trou et c'est un lift pour un autre temps. Tout change. On ne sait plus par coeur The Celebration of the Lizard.

C'est dans l'instant que tu es le plus à ta place. Tu récites Good times bad times dont la musique ne te revient pas. Firth of fifth t'échappe aussi. Sur la grève un chien erre comme du vent rendu visible. Here comes the sun revient à la surface et replonge en toi avec tout le reste. II fait encore soleil mais à moitié, seulement. Ne me quitte pas, dis, The way we were? Si tu aimes mieux, dis, Fool that i am? Je t'aime, je t'aime, et c'est pop. 


\section{ALORS}

alors $^{*}$ alors une fois de plus le désir* son plaisir et sa chute ${ }^{*}$ je veux dire ${ }^{*}$ comment $^{*}$

sa zizanie*

au coeur de tout ${ }^{*}$ droit au coeur ${ }^{*}$ de quelque nature que ce soit* me faire dans tout ça* être là où ça compte* de tous les côtés* peut-être ${ }^{*}$ mon verbe ${ }^{*}$ ma langue ${ }^{*}$ ma magie* me reçoivent* toute chair*

et cela $m^{\prime} a-t$-on dit fait du bien*

alors* alors* c'est dans les gestes que j'apprends le mieux de même que dans le beau vertige de toute une vie* je vois*

ma zizanie*

pas de discernement dans tout ca* simplement un corps* car $^{*}$ il y aura toujours un temps pour chaque chose ${ }^{*} c^{\prime} e s t$ voulu*

et cela c'est justement un mouvement*

alors* I'amour fou me sentant analphabète* possible tout de même* tout le reste c'est un renoncement au désir ${ }^{*}$ une mise de pur esprit*

deviennent matière mes dires pas ma chair* du moins l'autre chair* malgré tout* celle qui ne fait pas le trottoir ${ }^{*}$ mais à tout prix *

sans critère*

car rien n'est assis en moi* ni même dans le monde* ni même dans la nuit* tout bande* ${ }^{*}$ 'est la qualité $d^{\prime} e x i s t e r^{*}$ enfin pardelà ${ }^{*}$

la zizanie*

jusqu'au point de non retour*

et cela m'a-t-on dit c'est la fameuse dimension*

celle de l'exil* à travers un rapprochement de la peau* dans ce qui a toujours été là ${ }^{*}$ été comme été* ce qui me mange* à fond* et cela $m^{\prime} a-t$-on dit c'est le travail souterrain de la tête* dans laquelle tout est inné* permis* 
chaque coup que je porte au hasard me livre le désir et me fait désirer ${ }^{*}$ dans mon moi il y a ce bond de la bête*

alors* c'est ainsi que je pose la question* l'éclipse de ses prétextes*

je me suppose en chute*

car dans tout ça il y a la volonté de la différence à heure fixe* dans le temps du thème charne/* il y a la capacité jubilatoire* ce qui fait le nerf content* la révélation*

et tout cela m'a-t-on dit mène à l'eupho* rie*

à l'objet autour duquel j'espère ${ }^{*}$ une réplique ${ }^{*}$ un $i^{*}$ ci $^{*}$ suivant tout ce qui en moi est pris de failles et d'incréé* or nul ne saurait me guider là où je veux exactement aller*

et moi je n'ai pas à penser où je veux aller*

je me laisse porter*

par une voix*

qui s'appelle ma voix*

par un désir qui continue depuis l'éternité*

qui peut me sauver*

sans jugement ${ }^{*}$ sans mimétisme ${ }^{*}$ sans livre du devoir*

car rituellement je participe avant tout au corps*

à sa blessure ${ }^{*}$

à sa démesure où je veux la paix ${ }^{*}$ ce qui n'est pas dit* sûr* car enfin je crois trop en la notion d'amour* 